\title{
The Silicon Pixel Detector of the NA60 Experiment
}

\author{
M. Floris ${ }^{\mathrm{a}}$, K. Banicz ${ }^{\mathrm{b}}$, A. David ${ }^{\mathrm{c}}$, J.M. Heuser ${ }^{\mathrm{d}}$, M. Keil ${ }^{\mathrm{eb}}$, \\ C. Lourenço ${ }^{\mathrm{e}}$, H. Ohnishi ${ }^{\mathrm{d}}$, E. Radermacher ${ }^{\mathrm{e}}$, R. Shahoyan ${ }^{\mathrm{c}}$, G. Usai $^{\mathrm{a}}$ \\ ${ }^{a}$ Università degli Studi di Cagliari and INFN Cagliari, Italy \\ ${ }^{\mathrm{b}}$ Universtität Heidelberg, Heidelberg, Germany \\ ${ }^{\mathrm{c}}$ CFIF, Istituto Superior Técnico, Lisbon, Portugal \\ ${ }^{\mathrm{d}}$ RIKEN - The Institute of Physical and Chemical Research, Wako, Saitama, Japan \\ ${ }^{\mathrm{e}} \mathrm{CERN}$, Geneva, Switzerland
}

The NA60 experiment at the CERN SPS studies dimuon production in nucleus-nucleus and proton-nucleus collisions. The NA60 experimental program requires accurate tracking in the vertex region. In particular, it is crucial to distinguish between dimuons coming from the interaction vertex and muon pairs coming from the decay of charmed mesons. The high multiplicity of charged tracks produced in heavy-ion collisions imposes the use of radiation tolerant silicon pixel detectors. The NA60 silicon vertex detector is made of 16 planes, providing 11 tracking points. It was commissioned in the summer of 2003 and used in a 40-day-long data taking period in October/November 2003. This paper describes the detector, its performance and results from the 2003 IndiumIndium run.

\section{INTRODUCTION}

NA60 is a fixed-target experiment at the CERN SPS that studies dimuon production in protonnucleus and nucleus-nucleus collisions [1-3].

Its detector complements the muon spectrometer and zero degree calorimeter previously used in the NA50 experiment with a new vertex region using two state-of-the-art silicon detectors: a cryogenic silicon beam tracker and a silicon pixel telescope.

The main limiting factor in previous experiments comes from the hadron absorber of the muon spectrometer. It is needed to ensure that only muons can trigger the experiment, but, because of multiple scattering and energy loss, it limits the detector's dimuon mass resolution and renders accurate vertexing impossible. To overcome these limitations, NA60 needs a vertex tracker compatible with the high multiplicity environment of heavy-ion collisions and the high luminosities necessary to achieve reasonable dimuon statistics.
A tracker to be used in such environment must be both highly granular and radiation tolerant, a combination which only recently has become available with the development of radiation tolerant silicon pixel detectors.

Tracking in the vertex region significantly improves the mass resolution and the signal to noise ratio. The former by matching muon tracks before and after the absorber and the latter by rejecting muons from $\pi$ and $K$ decays, the main source of background.

The NA60 silicon pixel vertex detector enables measurements that would not have been possible only a few years ago.

For the first time in a fixed target heavy-ion experiment, it has also become possible to distinguish between muon pairs originating from the simultaneous semi-leptonic decays of $D$ mesons and dimuons coming from the interaction vertex, by measuring the offset of the tracks with respect to the interaction point. 


\section{THE SILICON PIXEL TELESCOPE}

The silicon pixel telescope consists of 16 physical planes providing 11 tracking points. The planes are made from assemblies of a silicon sensor chip bump-bonded to a radiation tolerant readout chip. The chips have a $256 \times 32$ matrix of $50 \times 425 \mu \mathrm{m}^{2}$ cells covering a sensitive area of $12.8 \times 13.6 \mathrm{~mm}^{2}$.

The ALICE1LHCb [4] readout chip is manufactured in a $0.25 \mu \mathrm{m}$ CMOS technology and designed with special techniques in order to increase its radiation tolerance. It has been shown to remain fully functional after an absorbed dose of about 12 Mrad [5].

The assemblies are tested with charge injections and radioactive source scans at the probe station in order to select good ones. An assembly is considered good if it has at least $96 \%$ working pixels.

Once selected, assemblies are glued on an $\mathrm{Al}_{2} \mathrm{O}_{3}$ or $\mathrm{BeO}$ ceramic hybrid, which is then mounted over an opening on a PCB, acting as the rigid frame of the plane and routing the readout lines out of the magnet gap. Electrical connection between the assemblies and the hybrid and between the hybrid and the PCB is assured with wire-bonds. The back side of the ceramic hybrid is fitted with a cooling ring.

The planes are cooled with water at $10{ }^{\circ} \mathrm{C}$, in order to maintain the sensors at a temperature below $30{ }^{\circ} \mathrm{C}$.

Each plane has a material budget of about $3 \% X_{0}$, mainly due to the silicon of the sensor $(300 \mu \mathrm{m})$ and readout chip $(750 \mu \mathrm{m})$ and to the gold used in the ground and power planes of the hybrid (effective thickness $\sim 30 \mu \mathrm{m}$ ).

The layout of the complete telescope is shown in Fig. 1. For acceptance reasons it is composed of planes of 2 different sizes: planes closer to the target are smaller. The small planes are composed of 4 chips, the large ones require 16 chips to cover the whole angular acceptance. For technical reasons they are arranged on two 8-chip physical modules.

The layout of the 8-chip planes is such that if one of two modules is turned upside down, and two planes are superimposed, the whole angular

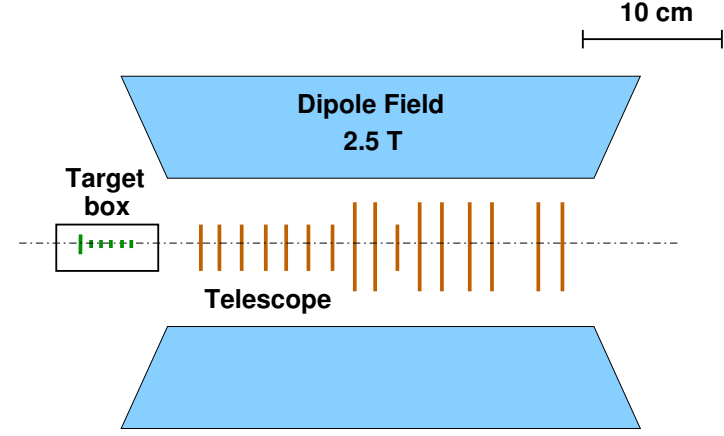

Figure 1. Layout of the NA60 silicon vertex telescope.

acceptance is covered. In this way the number of different plane layouts is minimized.

The whole telescope is placed inside a $2.5 \mathrm{~T}$ dipole field in order to measure particle momenta in the vertex region.

According to the orientation of the assemblies with respect to the magnetic field, the planes can provide the best resolution in the bending or in the non-bending plane.

Five small planes provide the best resolution in the bending plane, three small planes perpendicular to it: in this way the offset of the tracks with respect to the interaction vertex can be measured with a good resolution on all azimuthal angles.

All the large planes provide the best resolution in the bending plane, as they are mainly used to measure track curvature.

\section{READOUT ELECTRONICS}

The readout electronics of NA60 is based on the PCI architecture, profiting from its high bandwidth and ubiquity in commodity PCs. This represents increased performance at a fraction of the cost as, e.g., compared to VME. Additionally, interfacing the DAQ software is very easy, as PCI is natively supported by all modern operating systems.

The NA60 PCI readout system is modular. The basic readout element is a general purpose PCI card, common to all detectors, the PCI- 
CFD [6]. This card is interfaced to the different detector front-ends through specialized mezzanine cards [7] directly plugged on the CFD. This way the DAQ PC always sees the same interface, regardless of the underlying detector.

Each pixel detector plane is interfaced to its readout mezzanine by an adapter card. This is directly plugged on the pixel plane and contains two radiation tolerant chips: the pilot chip [8], also developed by the ALICE collaboration, and the GOL chip [9], developed at CERN.

During readout, the pilot chip gets the data from the pixel readout chips and sends them to the mezzanine through the GOL chip, over a GLink high-speed serial link. Columns are read out in parallel and chips on the same bus sequentially. The ALICE1LHCb chip is clocked at $10 \mathrm{MHz}$, so the readout time of an 8-chip plane is about $200 \mu \mathrm{s}$. The maximum occupancy of the pixel telescope is about $3 \%$, implying a maximum event size of $18 \mathrm{kB}$.

Configuration is provided to the readout and pilot chips via a JTAG interface by the mezzanine, over LVDS lines.

\section{RESULTS FROM DATA TAKING PE- RIODS}

The first three small planes were tested in October 2002 with a low energy Lead beam (20 and $30 \mathrm{AGeV}$ ). A detailed report of detector performance during this run can be found in [10].

In October and November 2003 the full telescope (16 planes) was operated in a 40-day-long run with a Indium beam at $158 \mathrm{AGeV}$ [11].

The results discussed in this section are obtained with a small sub-sample of the total statistics (less than 1\%) collected during this run.

The high levels of radiation during the high energy Indium run led to significant modifications in the detector behavior. Changes in the leakage current and in the depletion voltage were monitored throughout the run. Fig. 2 depicts the leakage current as a function of time. The open symbols show the measured leakage current, the filled ones the current corrected for a temperature of $20^{\circ} \mathrm{C}$.

The most exposed regions of the sensors under-

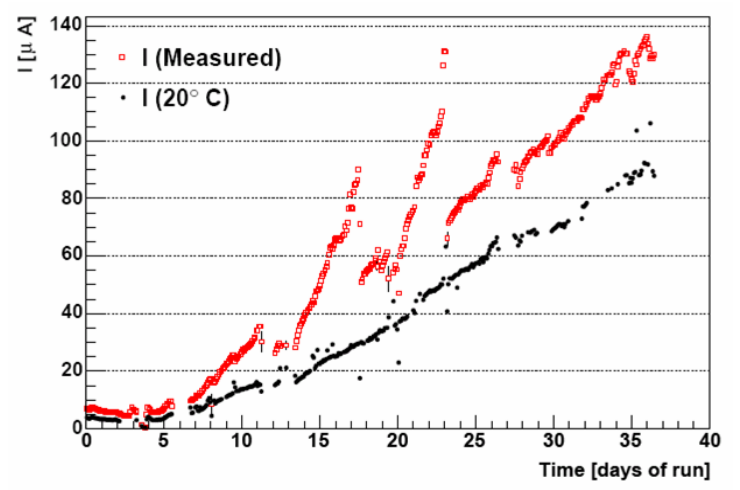

Figure 2. Leakage current as a function of time.

went type inversion. In accordance with the predictions of Monte Carlo studies, the detector remained fully operational throughout the run and the modifications in behavior agree with expectations. Details can be found in [12].

The vertex resolution is better than $200 \mu \mathrm{m}$ in the longitudinal coordinate and about $20 \mu \mathrm{m}$ in the transverse ones. Fig. 3 shows a $\mathrm{Z}$ vertex distribution: the 7 Indium targets are clearly seen between the target box windows.

The mass resolution at the $\omega$ peak is about $20 \mathrm{MeV}$ (Fig. 4, taken from [2]), in contrast with $\sim 80 \mathrm{MeV}$ obtained without the vertex detector.

\section{CONCLUSIONS}

A silicon vertex detector based on radiation tolerant silicon pixel detectors has been built and successfully operated during a 5-week-long physics run with an Indium beam of $158 \mathrm{AGeV}$.

A preliminary analysis of a very small fraction of the data has proved that the detector has the performance necessary to complete the broad and ambitious physics program of NA60.

\section{ACKNOWLEDGMENTS}

The authors would like to thank all the people that helped realizing the silicon pixel telescope.

This detector has been developed within the framework of the NA60 experiment. It is there- 


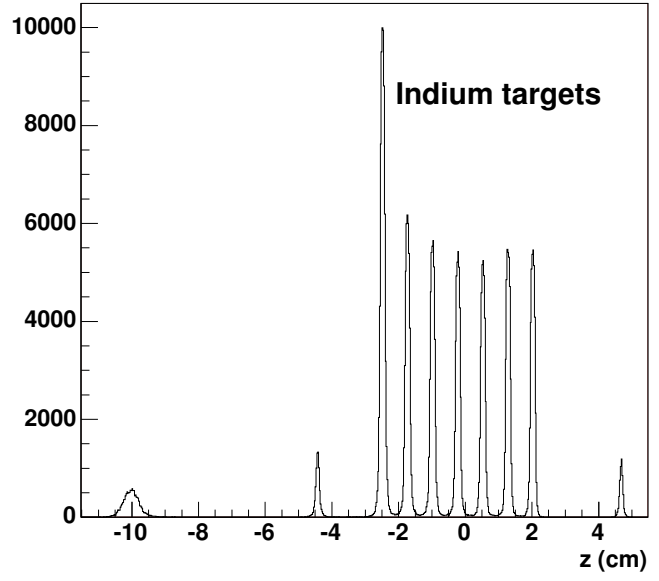

Figure 3. Z coordinate of the interaction vertex, as measured by the vertex telescope.

fore natural to start by thanking the colleagues from NA60.

The authors also thank the CERN EP/ED, $\mathrm{EP} / \mathrm{MIC}, \mathrm{EP} / \mathrm{TA} 1$ and EST/DEM groups, and the ALICE pixel project team, for their crucial contributions to the development of the telescope.

\section{REFERENCES}

1. NA60 Proposal, SPSC / P 316, May 15, 2000

2. P. Sonderegger et al. (NA60 Coll.), Proc. The Seventeenth International Conference on Ultra-Relativistic Nucleus-Nucleus Collisions (Quark Matter 2004), Oakland, California, USA, January 11-17, 2004

3. A. David et al. (NA60 Coll.), Proc. The Seventeenth International Conference on Ultra-Relativistic Nucleus-Nucleus Collisions (Quark Matter 2004), Oakland, California, USA, January 11-17, 2004

4. K. Wyllie et al., Proc. $5^{\text {th }}$ Workshop on Electronics for LHC Experiments, Snowmass, Colorado, USA, September 20-24, 1999.

5. J. Van Hunen, et al., "Irradiation and SPS Beam Tests of the Alice1LHCb Pixel Chip", CERN-ALI-2001-015.

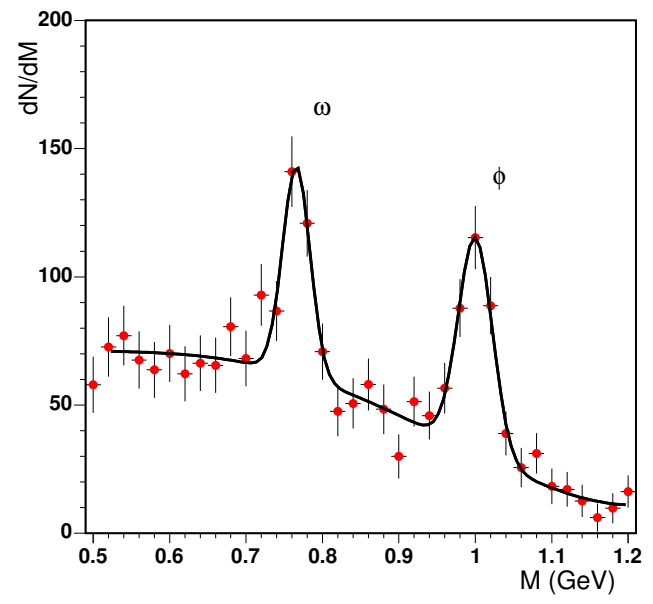

Figure 4. Dimuon invariant mass distribution [2].

6. M. Floris et al., Proc. 2003 IEEE Nuclear Science Symposium, Portland, Oregon, USA, October 19 - 25, 2003, submitted for publications on IEEE Trans Nucl Sci.

7. M. Floris et al., Proc. $13^{\text {th }}$ IEEE Real Time Conference, Montreal, Canada, May 18 - 23, 2003, to be published on IEEE Trans Nucl Sci.

8. A. Kluge for the Alice Collaboration, Proc. PIXEL 2002 workshop, Carmel, Sept. 2002.

9. P. Moreira et. al., Proc. Fifth Workshop on Electronics for LHC Experiments, Snowmass, Colorado, USA, 20-24 September 1999, pp. 194-198

10. M. Keil et al., "Operation and Performance of the NA60 Silicon Pixel Telescope", CERNPH-EP-2004-021, submitted for publication in Nucl. Instrum. Meth. A.

11. E. Radermacher for the NA60 Collaboration, Proc. 2003 IEEE Nuclear Science Symposium, Portland, Oregon, USA, October 19 25, 2003.

12. M. Keil et al. "Studies of irradiation effects in the NA60 silicon pixel detector", in preparation. 